\title{
Severe Acute Respiratory Syndrome Coronavirus Associated Myocarditis
}

\author{
Frans E. N. Wantania ${ }^{1 *}$, Ribka E. Wowor ${ }^{2}$, Ridwan Tandiawan ${ }^{1}$ \\ ${ }^{1}$ Department of Internal Medicine, Faculty of Medicine, Sam Ratulangi University, Prof. dr. R. D. Kandou Hospital, Manado, \\ Indonesia; ${ }^{2}$ Department of Public Health, Faculty of Public Health, Sam Ratulangi University, Manado, Indonesia
}

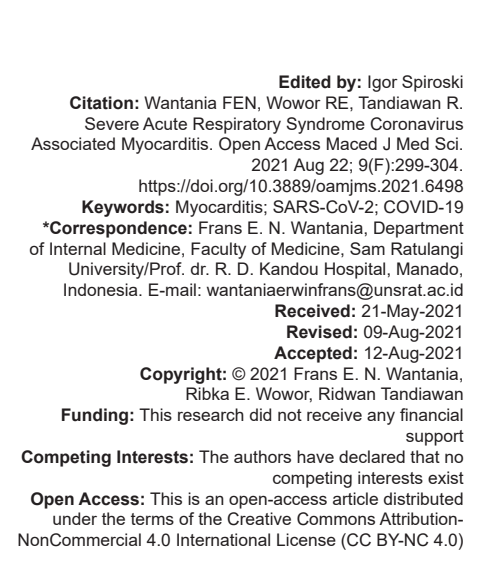

\begin{abstract}
Myocardial injury is common in patients with coronavirus disease 2019 (COVID-19). Among COVID-19 related myocardial injuries, etiology may vary, including myocarditis, myocardial infarct, sepsis-associated myocardia injury, and/or stress-induced cardiomyopathy. More data from prospective cohorts and case series are needed to understand the exact mechanism of COVID-19 associated myocardial injuries. It is clinically suspected that myocarditis is the cause of myocardial injury. However, myocarditis has a heterogeneous clinical presentation and tends to be underdiagnosed in critically ill COVID-19 patients. Due to the potential of rapid deterioration in the patient's condition, it is imperative to recognize myocarditis as a sequel to COVID-19, and a multidisciplinary team should be formed for managing all clinically suspected patients with COVID-19 associated myocarditis. Further studies are needed to recognize better and understand the relationship between myocarditis and COVID-19.
\end{abstract}

\section{Introduction}

Coronavirus disease 2019 (COVID-19) has been declared as a global pandemic by the World Health Organization on the March 11, 2020, because of how fast the virus spread throughout the world. Clinical presentation may vary, but most patients have respiratory tract symptoms. One study on 1099 COVID-19 patients showed that about $19 \%$ of patients had dyspnea, $41 \%$ needed oxygen supplementation, and $2.3 \%$ needed mechanical ventilation [1]. If not appropriately treated, COVID-19 can cause complications such as acute respiratory distress syndrome and multi-organ failure [2].

COVID-19 has a broad spectrum of cardiovascular complications, including acute heart failure, arrhythmia, acute coronary syndrome, myocarditis, and cardiac arrest. Myocarditis is one of the life-threatening complications observed among current and previous coronaviridae outbreaks, such as Middle East Respiratory Syndrome (MERS) [3]. Due to the high incidence report of myocarditis related to COVID-19, current data about COVID-19 and myocarditis relation are still being updated. Myocardium injury represents a rather significant mortality rate, reaching up to $51 \%$ when cardiac complications are involved [4].
COVID-19 associated myocarditis cases have been reported. The pathophysiology of COVID-19 related myocarditis is thought to be a combination of direct viral injury and cardiac damage due to the host's immune response. When a diagnosis is difficult to establish, an endomyocardial biopsy (EMB) can help identify heart infection through virus genome amplification. However, the long-term effects of COVID-19 myocarditis are still unknown [5]. The following review article will discuss severe acute respiratory syndrome coronavirus 2 (SARS-CoV-2) associated myocarditis.

\section{Viral Myocarditis: Definition, Epidemiology, and Etiology}

Myocarditis is defined as inflammation of the myocardium. The gold standard for diagnosing myocarditis is the Dallas criteria, which includes histopathology from the EMB. Dallas criteria have a low sensitivity for viral myocarditis because it does not detect the virus genome in the heart. In addition, to obtain a myocardium sample, an invasive procedure is needed [6]. 
Many viruses have been identified as the cause of myocarditis. Among these viruses, the most commonly encountered are adenoviruses and enteroviruses, including coxsackieviruses [6]. It was also reported that parvovirus B19 was associated with a significant percentage of patients diagnosed with myocarditis and dilated cardiomyopathy (DCM). However, some data suggested that parvovirus was also found in patients without myocarditis [6]. Viruses can cause myocarditis through the cellular and molecular mechanism, which has been investigated in several studies [7].

SARS-CoV-2 associated myocarditis is a rare occurrence, but myocardial injury appears to be more common in patients infected with COVID-19 than other types of coronavirus [8]. The prevalence rate of myocarditis in COVID-19 patients is still unclear. This is mainly because specific diagnostic modalities do not support the initial reports to assess myocarditis. Some experts said that up to $7 \%$ of COVID-19 related deaths are caused by myocarditis [9]. However, this is not supported by a confirmatory diagnosis of myocarditis, and thus a higher-than-expected number could be obtained. One study has identified that $24.5 \%$ of COVID-19 patients had coinfection with other types of virus [9].

\section{Pathology and Pathophysiology of Myocarditis in COVID-19}

The pathological examination can help determine the cause of myocardial injury, whether it is caused by systemic cytokines or direct infection of cardiomyocytes by the virus or other mechanisms. SARS-CoV-2 entry route is mediated by angiotensinconverting enzyme 2 and can cause acute cellular injury through cardiomyocyte, pericyte, or fibroblast infection that will lead to virus replication stage. Analysis of SARS-CoV-1 histological specimens has shown proof of direct viral infection in the myocardium and cells in the cardiac conduction system [10].

The previous evidence in acute myocarditis cases has shown that cellular injury is related to a combination of cardiotropic virus entry pathways and innate immune response, leading to diffuse, or focal myocardial necrosis. In COVID-19 infection, myocardial injury can potentially manifest as a sudden decline in clinical features in just a few days. Cardiotropic viruses such as SARS-CoV-1 have been found to disappear from the myocardium in about 5 days. However, some viruses can persist and survive in the myocardium for weeks to months, this is rare [4]. Assuming that SARS-CoV-2 can directly infect the myocardium, myocarditis incidence will manifest, especially during the acute or subacute phase. It is currently uncertain whether SARS-CoV-1 or SARS-CoV-2 is responsible for inducing antibody production due to molecular mimicry, as previously shown in coxsackie B protein virus interaction with the S2 area of the myocardium. The inflammation mechanism of SARS-CoV-2 can also cause chronic DCM. Myocardial injury is suspected to result from an increase in nitric oxide, which inhibits cardiomyocyte's response to calcium and decreases cardiac b1-adrenergic receptor regulation. However, the underlying mechanism is still not fully understood [11] (Figure 1).

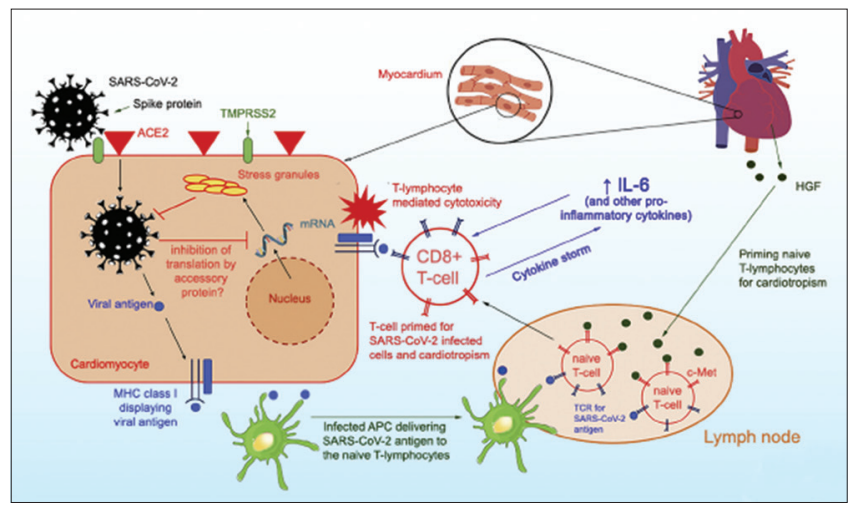

Figure 1: Pathophysiology of severe acute respiratory syndrome coronavirus 2 myocarditis

The activation of inflammatory cytokines can also cause myocardial injury in COVID-19 cases. Based on the serial autopsy report from three patients with COVID-19, it is described that SARS-CoV-2 was present in alveolar tissues but not in cardiac tissues. However, degenerative processes and necrosis suggested a secondary mechanism of injury. This raises the possibility that SARS-CoV-2, as previously described, might not directly cause cellular injury but through other mechanisms. In the context of immune system regulation and activation, acute myocardial injury is mediated by activated $\mathrm{T}$ and $\mathrm{B}$ cells, which trigger inflammation cascade, cytokine production, and antibody production [12].

Inflammatory response with cytokine production usually occurs in hospitalized patients with severe COVID-19 symptoms. This inflammation response may cause disseminated intravascular coagulopathy. One study in 183 COVID-19 patients in China showed that coagulopathy is associated with a higher mortality rate, and 15 out of 21 (71\%) patients passed away, which met the criteria for disseminated intravascular coagulopathy. Localized arterial pulmonary thrombosis in patients with the SARS and pulmonary embolism has been reported in COVID-19. Therefore, complications of microvascular thrombosis of the coronary blood vessel caused by disseminated intravascular coagulopathy are a potential but unproven mechanism that contributes to myocardial injury [10].

Observation on COVID-19 patients showed that there is an increase in pro-inflammatory cytokine that is known as cytokine storm. This cytokine storm is considered the basic pathogenesis mechanism of 
acute lung injury that amplifies immune response in alveolar tissues. The retrospective study by Chen et al. showed a similar result in which there was an increase in interleukin (IL)-2R, IL-6, IL-10, and tumor necrosis factor among patients with severe COVID-19. Cytokine storm incidence in severe COVID-19 is based on the fact that MERS-CoV and SARS-COV have shown increased cytokines among the spectrum of coronavirus disease, but whether this is the main cause of fulminant myocarditis remains uncertain [4].

SARS-CoV-2 infection may trigger arrhythmia through several mechanisms, including direct injury in cardiomyocyte that causes disturbance in the plasma membrane and electrical conductivity, a pericardial infection that leads to massive edema, ischemia due to microvascular disease caused by pericytes infection, arrhythmia caused by fibrosis or scarring of the myocardium, and pro-inflammatory cytokine that acts as arrhythmogenicity predisposition factor. Arrhythmia can occur during acute or chronic myocarditis and even after the patient has recovered. Several pro-inflammatory cytokines such as IL-6 can cause displacement of plakoglobin, a desmosomal protein, from the cardiomyocyte membrane. Reduced expression of the surface desmosomal protein is an etiology known as arrhythmogenic cardiomyopathy. Current evidence showed an increased level of serum IL-6 in COVID-19 patients, especially in patients with severe infection. Genetic predisposition appears to also play a role to some extent in inducing arrhythmia in SARS-CoV-2 infection [5], [13] (Figure 2).

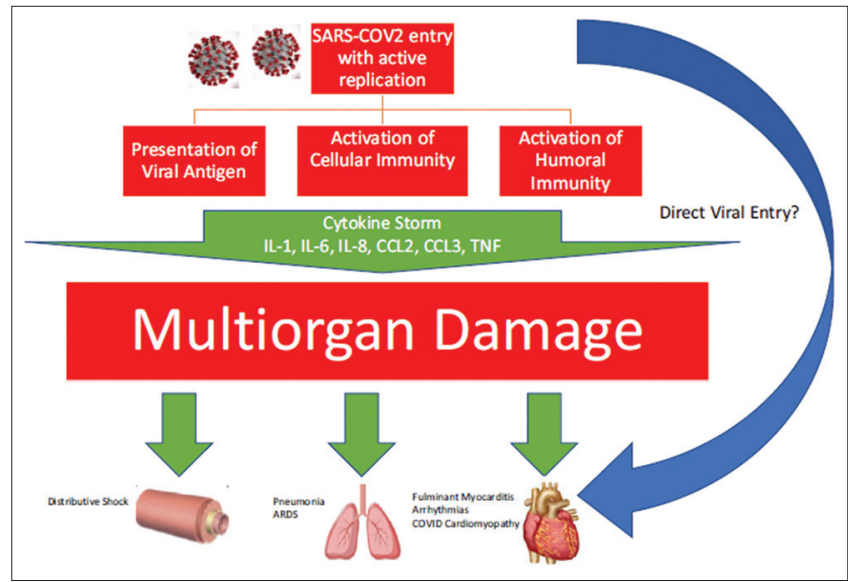

Figure 2: Pathogenesis mechanism and systemic effect of coronavirus disease 2019 that leads to multi-organ damage [4]

\section{Clinical Manifestation}

The clinical manifestations of SARS-CoV-2 myocarditis are various. Some patients showed relatively mild symptoms such as fatigue and dyspnea, and others reported chest pain or chest tightness during activity. Many patients deteriorated quickly and showed signs of tachycardia and acute onset heart failure with cardiogenic shock. In severe cases, patients may present signs of right heart failure, including increased jugular venous pressure, peripheral edema, and right upper quadrant abdominal pain. The most common presentation is fulminant myocarditis, which is defined as ventricular dysfunction and heart failure within 2-3 weeks after being infected with the virus. Early signs of fulminant myocarditis usually resemble sepsis signs, such as fever with a low pulse, cold extremities, or the presence of petechiae and sinus tachycardia [14].

Myocarditis may also present as sequelae after COVID-19 infection. However, this rarely happens. Reported cases of myocarditis in COVID-19 patients showed various clinical presentations. There is a potential for overlapping symptoms in patients with primary COVID-19 infection and COVID-19 patients with suspected clinical myocarditis [4].

A significant increase in serum troponin has been observed in several cases of severe COVID-19, which indicates cardiac involvement. A study by Zhou et al. demonstrated that troponin I has significantly higher sensitivity on the $4^{\text {th }}$ day in COVID-19 patients that did not survive compared to those who survived (8.8 pg/ $\mathrm{ml}$ vs. $2.5 \mathrm{pg} / \mathrm{ml}$ ). Myocardial injury can serve as a predictor for mortality and increasing severity of COVID19 patients [15]. However, isolated troponitis is not a part of myocarditis diagnosis and requires additional examination. Furthermore, troponin increase in severe COVID-19 can reflect the lack of oxygen condition resulting in myocardial injury. Other biomarkers, such as natriuretic peptide, are neither sensitive nor specific in diagnosing myocarditis [14].

Electrocardiogram (ECG) usually showed some abnormality in patients with myocarditis. However, this specific abnormality lacks sensitivity and specificity. Changes in ECG have been documented before in severe COVID-19 patients. He et al. reported 2 cases of ECG changes in hospitalized COVID-19 patients. In one of the cases, the initial ECG of the patient showed sinus rhythm with $1^{\text {st }}$ degree atrioventricular (AV) block, and 2 days later, the ECG showed $3^{\text {rd }}$ degree AV block. Nevertheless, dynamic ECG changes in COVID-19 patients may be a sign of clinical decline and should be closely monitored in all hospitalized COVID19 patients [16].

\section{Differential Diagnosis}

In the context of elevated troponin levels, acute coronary syndrome should be considered as a differential diagnosis. The epicardial disease can be confirmed with coronary angiography. However, many COVID-19 patients have been reported to have detectable levels of cTnl, even when they have no 
apparent cardiac symptoms. This finding usually does not stem from myocardial infarct. There is a possibility that the increase of troponin level is caused by exacerbation of subclinical coronary artery disease in patients with sepsis, which increases cardiac oxygen demand. This will cause a further imbalance between oxygen supply and demand that will eventually lead to myocardial infarct. Serial cardiac biomarker examination can help detect injury in the myocardium, especially if there is an increase in the biomarker [17].

During the recent decade, cardiovascular magnetic resonance (CMR) has become the diagnostic tool of choice for patients with evidence for acute nonischemic myocardial injury. Suspected myocarditis is one of the most frequent indications for CMR scans. CMR allows for targeting several features of myocarditis, including inflammatory hyperemia and edema, necrosis/scar, contractile dysfunction, and accompanying pericardial effusion can all be visualized during a single scan. Of note, the presence of necrosis or scar is a strong predictor of prognosis in patients with myocarditis [18].

One case series study showed that $67 \%$ of severe COVID-19 patients needed vasopressor, and about $33 \%$ have precipitated cardiomyopathy. This raises the suspicion that cardiomyopathy is associated with sepsis. Cardinal symptoms of sepsis-associated cardiomyopathy are dilatation of the left ventricle, ejection fraction disorders, and recovery within 7-10 days [5].

\section{Management of Myocarditis in COVID-19}

Several randomized clinical trial data regarding myocarditis management and guideline recommendations are based on a consensus. Patients with suspected myocarditis associated with COVID-19 should be treated in accordance with current practice guideline recommendations for heart failure and arrhythmia. Heart failure is often encountered ( $>20 \%$ ) in hospitalized COVID-19 patients. However, it is unclear whether this is caused by a preexisting decrease of left ventricular function, new-onset myocarditis, or a combination of both [11].

Steroidsandothertypesofimmunosuppressants are not recommended in patients with myocarditis. The Myocarditis Treatment Trial is the largest randomized control trial (RCT) to study the effect of immunosuppression in patients with myocarditis. In this study, 111 patients with myocarditis were treated with a regiment of prednisone and azathioprine, prednisone and cyclosporine, and placebo. There was no statistical difference regarding left ventricular ejection fraction after 28 weeks [19].
Systemic steroids and immunosuppressants together are not recommended for COVID-19 patients because it may worsen lung injury. In this case, a monotherapy with immunosuppressant may seem beneficial for patients with cytokine storms. However, current ongoing clinical trials with treatment regimens including steroids, intravenous immunoglobulin, selective cytokine blocker (for example, anakinra, or tocilizumab), and JAK inhibitor showed no clear evidence of additional benefit compared to other supportive treatments. At present, there is not enough evidence to recommend systemic immunosuppression therapy in COVID-19 associated myocarditis [4].

Antiviral therapy has already been used in patients with virus myocarditis. However, this RCT has not determined specific virus as the cause of myocarditis, such as enterovirus or adenovirus, as the main target therapy. For example, in a randomized clinical trial of Betaferon in chronic viral cardiomyopathy, there was no increase in viral clearance for all virus subtypes. In this study, the patients were given either placebo or interferon-B, and the diagnosis was confirmed using EMB. At present, antiviral therapy is still being investigated for potential therapy in COVID-19. Lopinavir/ritonavir is a combination of antivirus that is commonly used to treat the Human Immunodeficiency Virus. This antivirus is currently undergoing clinical trial for COVID-19. One RCT showed that there was no difference in mortality rate between lopinavir/ritonavir and placebo. Furthermore, lopinavir/ritonavir could be associated with the increase of QT interval. To date, no antivirus therapy is known to be beneficial for COVID19 patients [20].

Supportive therapy is still the mainstay choice of treatment method, and this is due to the lack of evidence that combinations of immunosuppressive therapy can improve the condition of patients with myocarditis. Steroid monotherapy, steroid combination therapy with an IL-6 inhibitor, antiviral monotherapy without immunomodulation has all been used for COVID-19 associated myocarditis, but the effect of immunosuppression in treating fulminant myocarditis has yet been determined [21].

Patients with myocarditis may clinically deteriorate or develop end-organ failure despite being given heart failure and other supportive therapies. Before the COVID-19 pandemic, end-stage myocarditis nonCOVID-19 could be referred for mechanical circulatory support (MCS) and/or heart transplant consideration. MCS has successfully been proven to help recovery in patients with fulminant myocarditis. Zhang et al. reported a fulminant myocarditis case that successfully recovered using MCS with oxygenator membrane extracorporeal as an alternative treatment in COVID19 patients. Heart transplantation is not an option for the patient with COVID-19 associated myocarditis due to the active infection state [4], [22]. 


\section{Conclusion}

It is clinically suspected that myocarditis is the cause of myocardial injury. Myocarditis has a heterogeneous clinical presentation and tends to be underdiagnosed in critically ill COVID-19 patients. Due to the potential of rapid deterioration in the patient's condition, it is imperative to recognize myocarditis as a sequel to COVID-19, and a multidisciplinary team should be formed for managing all clinically suspected patients with COVID-19 associated myocarditis. Further studies are needed to recognize better and understand the relationship between myocarditis and COVID-19. Treatment for myocarditis in COVID-19 is still supportive, and there is no recommended therapy to date.

\section{References}

1. Lie SA, Wong SW, Wong LT, Wong TG, Chong SY Practical considerations for performing regional anesthesia: Lessons learned from the COVID-19 pandemic. Can J Anaesth. 2020;67(7):885-92. https://doi.org/10.1007/ s12630-020-01637-0

PMid:32212103

2. Arentz M, Yim E, Klaff L, Lokhandwala S, Riedo FX, Chong M, et al. Characteristics and outcomes of 21 critically ill patients with COVID-19 in Washington State. JAMA. 2020;323(16):1612-4. https://doi.org/10.1001/jama.2020.4326

PMid:32191259

3. Alhogbani T. Acute myocarditis associated with novel Middle East respiratory syndrome coronavirus. Ann Saudi Med. 2016;36(1):78-80. https://doi.org/10.5144/0256-4947.2016.78 PMid:26922692

4. Pirzada A, Mokhtar AT, Moeller AD. COVID-19 and myocarditis: What do we know so far? CJC Open. 2020;2(4):278-85. https:// doi.org/10.1016/j.cjco.2020.05.005

PMid:32691024

5. Siripanthong $B$, Nazarian $S$, Muser D, Deo R, Santangeli $P$, Khanji MY, et al. Recognizing COVID-19-related myocarditis: The possible pathophysiology and proposed guideline for diagnosis and management. Heart Rhythm. 2020;17(9):146371. https://doi.org/10.1016/j.hrthm.2020.05.001 PMid:32387246

6. Yajima T, Knowlton KU. Viral myocarditis: From the perspective of the virus. Circulation. 2009;119(19):2615-24. https://doi. org/10.1161/circulationaha.108.766022

PMid:19451363

7. Schenk T, Enders M, Pollak S, Hahn R, Huzly D. High prevalence of human parvovirus B19 DNA in myocardial autopsy samples from subjects without myocarditis or dilative cardiomyopathy. J Clin Microbiol. 2009;47(1):106-10. https://doi.org/10.1128/ jcm.01672-08

PMid: 19005147

8. $\mathrm{Hu} \mathrm{H}$, Ma F, Wei $\mathrm{X}$, Fang $\mathrm{Y}$. Coronavirus fulminant myocarditis treated with glucocorticoid and human immunoglobulin. Eur Heart J. 2021;42(2):206. https://doi.org/10.1093/eurheartj/

\section{ehaa190}

PMid:32176300

9. Driggin E, Madhavan MV, Bikdeli B, Chuich T, Laracy J, BiondiZoccai G, et al. Cardiovascular considerations for patients, health care workers, and health systems during the COVID-19 pandemic. J Am Coll Cardiol. 2020;75(18):2352-71. https://doi. org/10.1016/j.jacc.2020.03.031

PMid:32201335

10. Hendren NS, Drazner MH, Bozkurt B, Cooper LT Jr. Description and proposed management of the acute COVID-19 cardiovascular syndrome. Circulation. 2020;141(23):1903-14. https://doi.org/10.1161/circulationaha.120.047349 PMid:32297796

11. Zhou F, Yu T, Du R, Fan G, Liu Y, Liu Z, et al. Clinical course and risk factors for mortality of adult inpatients with COVID-19 in Wuhan, China: A retrospective cohort study. Lancet. 2020;395(10229):1054-62. https://doi.org/10.1016/ s0140-6736(20)30566-3

PMid:32171076

12. Tian S, Hu W, Niu L, Liu H, Xu H, Xiao SY. Pulmonary pathology of early-phase 2019 novel coronavirus (COVID-19) pneumonia in two patients with lung cancer. J Thorac Oncol. 2020;15(5):7004. https://doi.org/10.20944/preprints202002.0220.v2 PMid:32114094

13. Chen L, Li X, Chen M, Feng Y, Xiong C. The ACE2 expression in human heart indicates new potential mechanism of heart injury among patients infected with SARS-CoV-2. Cardiovasc Res. 2020;116(6):1097-100. https://doi.org/10.1093/cvr/cvaa078 PMid:32227090

14. Inciardi RM, Lupi L, Zaccone G, Italia L, Raffo M, Tomasoni D, et al. Cardiac Involvement in a patient with coronavirus disease 2019 (COVID-19). JAMA Cardiol. 2020;5(7):819-24. https://doi. org/10.1001/jamacardio.2020.1096 PMid:32219357

15. Metkus TS, Sokoll LJ, Barth AS, Czarny MJ, Hays AG, Lowenstein CJ, et al. Myocardial injury in severe COVID19 compared with non-COVID-19 acute respiratory distress syndrome. Circulation. 2020;143(6):553-65. https://doi. org/10.1161/circulationaha.120.050543

PMid:33186055

16. Caforio AL, Pankuweit S, Arbustini E, Basso C, GimenoBlanes J, Felix SB, et al. Current state of knowledge on aetiology, diagnosis, management, and therapy of myocarditis: A position statement of the European Society of Cardiology Working Group on myocardial and pericardial diseases. Eur Heart J. 2013;34(33):2636-48. https://doi.org/10.1093/ eurheartj/eht210

PMid:23824828

17. Clerkin KJ, Fried JA, Raikhelkar J, Sayer G, Griffin JM, Masoumi A, et al. COVID-19 and cardiovascular disease. Circulation. 2020;141(20):1648-55. https://doi.org/10.1161/ circulationaha.120.046941

PMid:32200663

18. Chu GC, Flewitt JA, Mikami Y, Vermes E, Friedrich MG. Assessment of acute myocarditis by cardiovascular MR: Diagnostic performance of shortened protocols. Int J Cardiovasc Imaging. 2013;29:1077-83. https://doi.org/10.1007/ s10554-013-0189-7

PMid:23404383

19. Tschöpe C, Cooper LT, Torre-Amione G, Van Linthout S. Management of myocarditis-related cardiomyopathy in adults. Circ Res. 2019;124(11):1568-83. https://doi.org/10.1161/ circresaha.118.313578

20. Cao B, Wang Y, Wen D, Liu W, Wang J, Fan G, et al. A Trial of 
lopinavir-ritonavir in adults hospitalized with severe COVID-19 N Engl J Med. 2020;382(19):1787-99.

PMid:32187464

21. Coyle J, Igbinomwanhia E, Sanchez-Nadales A, Danciu S,
Chu C, Shah N. A recovered case of COVID-19 myocarditis and ARDS treated with corticosteroids, tocilizumab, and experimental AT-001. JACC Case Rep. 2020;2(9):1331-6. https://doi.org/10.1016/j.jaccas.2020.04.025

PMid:32368755 